\title{
Molecular dynamics analysis of a rationally designed aldehyde dehydrogenase gives insights into improved activity for the non-native cofactor NAD ${ }^{+}$
}

Tobias J. Gmelch ${ }^{1}$, Josef M. Sperl' ${ }^{1}$, Volker Sieber ${ }^{1,2,3,4 *}$

${ }^{1}$ Chair of Chemistry of Biogenic Resources, Technical University of Munich, Campus Straubing for Biotechnology and Sustainability, Schulgasse 16, 94315 Straubing, Germany

${ }^{2}$ Catalysis Research Center, Technical University of Munich, Garching, Germany

3 Fraunhofer Institute of Interfacial Biotechnology (IGB), Bio-, Electro- and Chemo Catalysis (BioCat) Branch, Straubing, Germany

${ }^{4}$ School of Chemistry and Molecular Biosciences, The University of Queensland, St. Lucia, QLD, Australia

${ }^{*}$ Correspondence should be addressed to

Volker Sieber, Prof.

E-Mail: sieber@tum.de

Tel: +499421187301

\section{Supporting Information}

\section{Calculation of screening coverage}

Without further investigation, we assumed a yield of $50 \%$ for our mutagenesis approach. The codon-based coverage was then calculated applying the formula given below, with " $x$ " representing the colonies analyzed and "a" the amount of possible codons $(1 \mathrm{xNNK}=32) .{ }^{1}$

Codoncoverage $[\%]=1-\left(1-\frac{1}{a}\right)^{0.5 x} \cdot 100 \%$

Another approach to describe the coverage is to calculate the probability of the occurrence of the most active variants within the library. ${ }^{2}$ The probabilities $\mathrm{P}_{\mathrm{T} 1}$ and $\mathrm{P}_{\mathrm{T} 2}$, which correspond to the detection probability of the best or one of the two best variants of the library, were calculated by 
the online tool TopLib (http://trachel-srv.cs.haifa.ac.il/rachel/toplib/). A comparison to the codon coverage is shown in Supplemental Table 1.

Supplemental Table 1: Library coverage assuming a mutagenesis yield of $50 \%$

\begin{tabular}{lllll}
\hline & $\begin{array}{l}\text { Screened } \\
\text { variants }\end{array}$ & $\begin{array}{l}\text { Codon } \\
\text { coverage }\end{array}$ & $\mathbf{P}_{\mathrm{T} 1}$ & $\mathbf{P}_{\mathrm{T} 2}$ \\
\hline 1xNNK & $>80$ & $>72 \%$ & $>81 \%$ & $>96 \%$ \\
2xNNK & $>2500$ & $>71 \%$ & $>86 \%$ & $>98 \%$ \\
\hline
\end{tabular}

DNA-sequence of initial triple mutant TaALDH M0

Bold nucleotides correspond to the his-tag from the expression vector.

ATGGATACCAAACTGTATATTGATGGCCAGTGGGTTAATAGCAGCAGCGGTAAAACCGTTG ATAAATATTCTCCGGTTACCGGTCAGGTTATTGGTCGTATGGAAGCAGCAACCCGTGATGA TGTTGATCGTGCAATTGATGCAGCAGAAGATGCATTTTGGGCCTGGAATGATCTGGGTAGC GTTGAACGCAGCAAAATTATTTATCGTGCCAAAGAACTGATTGAAAAAAATCGTGCCGAACT GGAAAATATTATTATGGAAGAAAATGGCAAACCGGTGAAAGAAGCCAAAGAAGAAGTCGAC GGCGTCATTGATCAGATTCAGTATTATGCAGAATGGGCACGTAAACTGAATGGTGAAGTTG TTGAAGGCACCAGCAGCCATCGTAAAATTTTTCAGTATAAAGTGCCGTATGGTATTGTTGTT GCACTGACCCCGTGGAATTTTCCGGCAGGCATGGTTGCCCGTAAACTGGCACCGGCACTG CTGACCGGTAATACCGTTGTTCTGAAACCGAGCAGCGATACACCGGGTAGCGCAGAATGG ATTGTGCGTAAATTTGTTGAAGCCGGTGTTCCGAAAGGTGTGCTGAATTTTATTACCGGTCG TGGTAGCGAAATTGGCGATTACATTGTGGAACATAAAAAAGTCAATCTGATTACCATGACCG GTAGCACCGCAACAGGTCAGCGCATTATGCAGAAAGCAAGCGCAAATATGGCAAAACTGAT TCTGGAACTGGGTGGTAAAGCACCGTTTATGGTTTGGAAAGATGCCGATATGGATAATGCA CTGAAAACCCTGCTGTGGGCAAAATATTGGAATGCCGGTCAGAGCTGTATTGCAGCAGAAC GTCTGTATGTGCATGAAGATATTTATGATACCTTTATGAGCCGTTTTGTTGAACTGAGCCGC AAACTGGCACTGGGTGATCCGAAAAATGCAGATATGGGTCCGCTGATTAATAAAGGTGCAC TGCAGGCAACCAGCGAAATTGTTGAAGAAGCGAAAGAATCTGGCGCAAAAATTCTGTTTGG TGGTAGCCAGCCGAGCCTGAGCGGTCCGTATCGTAATGGCTATTTTTTTCTGCCGACCATT ATTGGTAATGCGGATCAGAAAAGCAAAATCTTTCAGGAAGAAATTTTTGCACCGGTTATTGG TGCACGTAAAATTAGCAGCGTGGAAGAAATGTGTGATCTGGCCAATGATAATAAATATGGTC TGGCCAGCTACCTGTTTACCAAAGATCCGAATATCATTTTTGAAGCCAGCGAACGTATTCGT TTTGGTGAACTGTATGTGAATATGCCGGGTCCGGAAGCAAGCCAGGGTTATCACACCGGTT TTCGTATGACAGGTCAGGCAGGCGAAGGTTCTAAATATGGCATTAGCGAATATCTGAAACT GAAAAATATTTATGTGGATTATAGCGGCAAACCGCTGCATATTAATACCGTTCGTGATGACC TGTTTCAGAGCGGGAGACCTGTGCTGGGCAGCAGCCACCACCACCACCACCACTAA

\section{Application of CSR-SALAD for identification of mutagenesis positions}

CSR-SALAD predicts cofactor specificity determining residues using crystal structures with bound native cofactor as input. ${ }^{3}$ We were aiming on enhancing the activity for the non-native cofactor $\mathrm{NAD}^{+}$and not necessarily on reversing the selectivity and thus used a model with docked $\mathrm{NAD}^{+}$as input for determining mutagenesis positions. A comparison of the CSR-SALAD 
output for $\mathrm{M0}\left(\mathrm{NAD}^{+}\right)$with $\mathrm{M0}\left(\mathrm{NADP}^{+}\right)$shows, that the highly conserved position $\mathrm{K} 172$, which is essential for ribose binding in $\mathrm{NAD}(\mathrm{P})$-dependent aldehyde dehydrogenases ${ }^{4}$, is suggested for both input structures. In case of the NADP ${ }^{+}$docked structure, L145 is suggested to be selectivity-determining instead of S174 and G205. Concerning the medium and low priority targets, both results overlap with V213 and E207 as additional targets for M0 (NADP ${ }^{+}$).

$\begin{array}{llll}\text { Residue } & \text { Type } & \text { Codon } & \text { AAs } \\ \text { LYS 172 } & \text { Nonsimple } & \text { AVK } & \text { KNRST } \\ \text { SER 174 } & \text { Pyrophosphate RSC } & \text { AGST } \\ \text { GLY 205 } & \text { Face } & \text { RSA } & \text { AGRT }\end{array}$

Suggested library size: 96

The following residues should be targeted first for activity-recovery by site-saturation mutagenesis:

Medium priority:

GLY 205

GLY 209

THR 229

ARG 232

ILE 233

Low priority:

ASP 176

ASP 210

\section{Selected Options:}

Maximum library size 850

Rossman fol d only: False

Exclude pyrophosphate-binding residues: False

Exclude peripheral residues: False

Verbose output: False

Supplemental Figure 1: CSR-Salad output for MO docked with NAD. 
Supplemental Table 2: Comparison of CSR-SALAD results depending on the type of cofactor docked within the input structure. Residues in italics are suggested for mutagenesis for both input structures.

\begin{tabular}{llll}
\hline $\begin{array}{l}\text { Docked } \\
\text { cofactor }\end{array}$ & $\begin{array}{l}\text { Selectivity } \\
\text { determining }\end{array}$ & $\begin{array}{l}\text { Medium } \\
\text { priority }\end{array}$ & $\begin{array}{l}\text { Low } \\
\text { priority }\end{array}$ \\
\hline NAD & Lys172 & Gly205 & Asp176 \\
& Codon: AVK & Gly209 & Asp210 \\
& Ser174 & Thr229 & \\
& Codon: RSC & Arg232 & \\
& Gly205 & Ile233 & \\
NADP & Lodon: RSA & & \\
& Cou145 & Asp210 & Asp176 \\
& Lys172 & Thr229 & Asp210 \\
& Codon: AVK & Arg232 & \\
& & Ile233 & \\
\hline
\end{tabular}


Supplemental Table 3: Result of Ramachandran analysis via RAMPAGE. Underlined residues are found in both structures.

\begin{tabular}{|c|c|c|}
\hline & $5 \mathrm{M} 4 \mathrm{X}$ & Model structure of M0 \\
\hline $\begin{array}{l}\text { Total residues } \\
\text { in structure }\end{array}$ & 461 & 493 \\
\hline $\begin{array}{l}\text { Allowed } \\
\text { region }\end{array}$ & $\begin{array}{l}\mathrm{G} 140, \underline{\mathrm{K} 251}, \mathrm{~N} 315, \\
\mathrm{E} 339, \mathrm{~S} 340, \underline{\mathrm{Y} 361}, \\
\underline{\mathrm{D} 404}, \underline{\mathrm{L} 409}, \mathrm{E} 466,\end{array}$ & $\begin{array}{l}\text { K92, W114, L118, T126, K136, V143, A237, } \\
\text { A239, K251, D261, Q279, A283, G311, E336, } \\
\underline{\text { Y361 }}, \mathrm{N} 370, \mathrm{I377}, \mathrm{S} 393, \underline{\mathrm{D} 404}, \underline{\mathrm{L} 409}, \mathrm{R} 429, \\
\mathrm{R} 451, \mathrm{M} 452, \mathrm{~A} 456, \mathrm{~S} 460\end{array}$ \\
\hline $\begin{array}{l}\text { Outlier } \\
\text { region }\end{array}$ & $\underline{\mathrm{G}} 408$ & P93, P178, P313, G408 \\
\hline
\end{tabular}

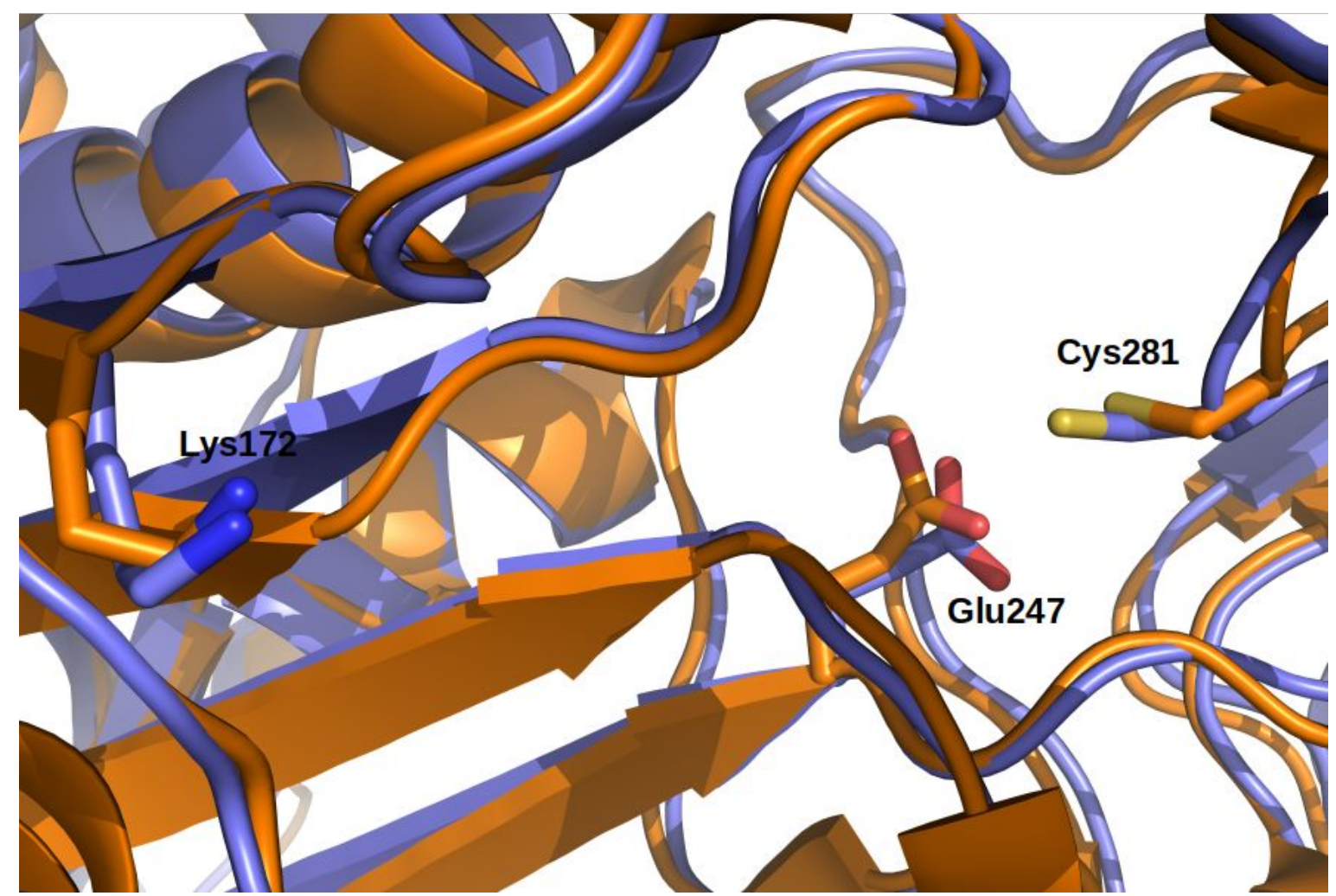

Supplemental Figure 2: Overlay of MO model (blue) with 2IMP crystal structure (orange). Position numbering based on TaALDH 
Supplemental Table 4: Typical site-directed mutagenesis protocol

\begin{tabular}{llll}
\hline Vol $[\boldsymbol{\mu L}]$ & Stock & compound & final conc. \\
\hline $\mathbf{1 0}$ & $5 \mathrm{x}$ & HF-Phusion buffer & $1 \mathrm{x}$ \\
$\mathbf{1}$ & $10 \mathrm{mM}$ & dNTPs & $0.2 \mathrm{mM}$ \\
$\mathbf{2 . 5}$ & $10 \mu \mathrm{M}$ & Each oligonucleotide & $0.5 \mu \mathrm{M}$ \\
$\mathbf{0 . 5}$ & $<300 \mathrm{ng} / \mu \mathrm{L}$ & DNA template & $<3 \mathrm{ng} / \mu \mathrm{L}$ \\
$\mathbf{0 . 5}$ & $2 \mathrm{U} / \mu \mathrm{L}$ & Phusion polymerase & $0.02 \mathrm{U} / \mu \mathrm{L}$ \\
$\mathbf{2 . 5}$ & $100 \%$ & DMSO & $5 \%$ \\
\hline
\end{tabular}

Supplemental Table 5: List of oligonucleotides. Bold letters indicate the modified position

\begin{tabular}{|l|l|l|l|}
\hline$\#$ & Pos & Name & 5'-3' sequence \\
\hline 1 & 172 & 172 NNK-f & GTAATACCGTTGTTCTGNNKCCGAGCAGCGATACAC \\
\hline 2 & 172 & 172 NNK-r & GTGTATCGCTGCTCGGMNNCAGAACAACGGTATTAC \\
\hline 3 & 174 & 174 NNK-f & GTTGTTCTGAAACGNNKAGCGATACACCGGGTAG \\
\hline 4 & 174 & 174 NNK-r & CTACCCGGTGTATCGCTMNNCGGTTTCAGAACAAC \\
\hline 5 & 175 & 175 NNK-f & GTTGTTCTGAAACCGAGCNNKGATACACCGGGTAGC \\
\hline 6 & 175 & $175-N N K-r$ & GCTACCCGGTGTATCMNNGCTCGGTTTCAGAACAAC \\
\hline 7 & 176 & 176 NNK-f & CTGAAACGAGCAGCNNKACACCGGGTAGCGC \\
\hline 8 & 176 & 176 NNK-r & GCGCTACCCGGTGTMNNGCTGCTCGGTTTCAG \\
\hline 9 & 177 & 177 NNK-f & GAAACCAGCAGCGATNNKCCGGGTAGCGCAGATG \\
\hline 10 & 177 & 177 NNK-r & CATTCTGCGCTACCCGGMNNATCGCTGCTCGGTTTC \\
\hline 11 & 205 & 205 NNK-f & GAATTTTATTACCGGTCGTNNKAGCGAAATTGGCG \\
\hline 12 & 205 & 205 NNK-r & CGCCAATTTCGCTMNNACGACCGGTAATAAAATTC \\
\hline 13 & 206 & 206 NNK-f & GAATTTTATTACCGGTCGTGGTNNKGAAATTGGCGATTAC \\
\hline 14 & 206 & 206 NNK-r & GTAATCGCCAATTTCMNNACCACGACCGGTAATAAAATTC \\
\hline 15 & 207 & 207 NNK-f & CCGGTCGTGGTAGCNNKATTGGCGATTACATTG \\
\hline 16 & 207 & 207 NNK-r & CAATGTAATCGCCAATMNNGCTACCACGACCGG \\
\hline 17 & 209 & 209 NNK-f & CGTGGTAGCGAAATTNNKGATTACATTGTGGAAC \\
\hline 18 & 209 & 209 NNK-r & GTTCCACAATGTAATCMNNAATTTCGCTACCACG \\
\hline 19 & 210 & 210 NNK-f & CGTGGTAGCGAAATTGGCNNKTACATTGTGGAAC \\
\hline 20 & 210 & 210 NNK-r & GTTCCACAATGTAMNNGCCAATTTCGCTACCACG \\
\hline 21 & 229 & 229 NNK-f & GACCGGTAGCACCGCANNKGGTCAGCGCATTATGC \\
\hline 22 & 229 & 229 NNK-r & GCATAATGCGCTGACCMNNTGCGGTGCTACCGGTC \\
\hline 23 & 232 & 232 NNK-f & GCACCGCAACAGGTCAGNNKATTATGCAGAAAGC \\
\hline 24 & 232 & 232 NNK-r & GCTTTCTGCATAATMNNCTGACCTGTTGCGGTGC \\
\hline 25 & 262 & $262-N N K-f$ & GGAAAGATGCCGATNNKGATAATGCACTGAAAACCC \\
\hline 26 & 262 & $262-N N K-r$ & GGGTTTTCAGTGCATTATCMNNATCGGCATCTTTCC \\
\hline 27 & 275 & 275 NNK-f & CCCTGCTGTGGGCAAAATATNNKAATGCCGGTCAGAGC \\
\hline 28 & 275 & $275-N N K-r$ & GCTCTGACCGGCATTMNNATATTTTGCCCACAGCAGGG \\
\hline 29 & 306 & 306 NNK-f & GTTTTGTTGAACTGAGCNNKAAACTGGCACTGGGTG \\
\hline
\end{tabular}




\begin{tabular}{|l|l|l|l|}
\hline 30 & 306 & 306 NNK-r & CACCCAGTGCCAGTTTMNNGCTCAGTTCAACAAAAC \\
\hline 31 & 349 & 349 NNK-f & CTGTTTGGTGGTNNKCAGCCGAGCCTGAGCGGTC \\
\hline 32 & 349 & 349 NNK-r & GACCGCTCAGGCTCGGCTGMNNACCACCAAACAG \\
\hline 33 & 271 & $271-N N K-f$ & GCACTGAAAACCCTGCTGNNKGCAAAATATTGGAATGCC \\
\hline 34 & 271 & $271-N N K-r$ & GGCATTCCAATATTTTGCMNNCAGCAGGGTTTTCAGTGC \\
\hline 35 & $271+$ & $271+275$ & CCCTGCTGNNKGCAAAATATNNKAATGCCGGTCAG \\
& 275 & NNK-f & \\
\hline 36 & $271+$ & $271+275$ & CTGACCGGCATTMNNATATTTTGCMNNCAGCAGGG \\
& 275 & NNK-r & \\
\hline 37 & 175 & S175E-f & GTTGTTCTGAAACCGAGCGAGGATACACCGGGTAGC \\
\hline 38 & 175 & S175E-r & GCTACCCGGTGTATCCTCGCTCGGTTTCAGAACAAC \\
\hline 39 & 176 & D176S-f & CTGAAACCGAGCAGCTCTACACCGGGTAGCGC \\
\hline 40 & 176 & D176S-r & GCGCTACCCGGTGTAGAGCTGCTCGGTTTCAG \\
\hline 41 & 206 & S206K-f & GAATTTTATTACCGGTCGTGGTAAGGAAATTGGCGATTAC \\
\hline 42 & 206 & S206K-r & GTAATCGCCAATTTCCTTACCACGACCGGTAATAAAATTC \\
\hline 43 & 262 & M262I-f & GGAAAGATGCCGATATTGATAATGCACTGAAAACCC \\
\hline 44 & 262 & M262I-r & GGGTTTTCAGTGCATTATCAATATCGGCATCTTTCC \\
\hline 45 & $175+$ & S175E + & CTGAAACCGAGCGAGTCTACACCGGGTAGCGC \\
& 176 & D176S-f & \\
\hline 46 & $175+$ & S175E + & GCGCTACCCGGTGTAGACTCGCTCGGTTTCAG \\
& 176 & D176S-r & \\
\hline
\end{tabular}

Supplemental Table 6: Amino acid at position 271 or 275 of the most active variant depending on the genetic background. All positions were mutated separately at either 271 or 275 and the two most active variants were sequenced, *M33 was mutated simultaneously at both positions

\begin{tabular}{lll}
\hline Template & W271 & W275 \\
\hline M0 & Y/L & T/L \\
S175E & W & N/T \\
D176S & $\mathrm{Y}$ & $\mathrm{S} / \mathrm{I}$ \\
S206K & $\mathrm{T} / \mathrm{N}$ & $\mathrm{L}$ \\
M262I & $\mathrm{L} / \mathrm{I}$ & $\mathrm{M} / \mathrm{L}$ \\
M33* $^{*}$ & $\mathrm{Y}$ & $\mathrm{V}$ \\
\hline
\end{tabular}




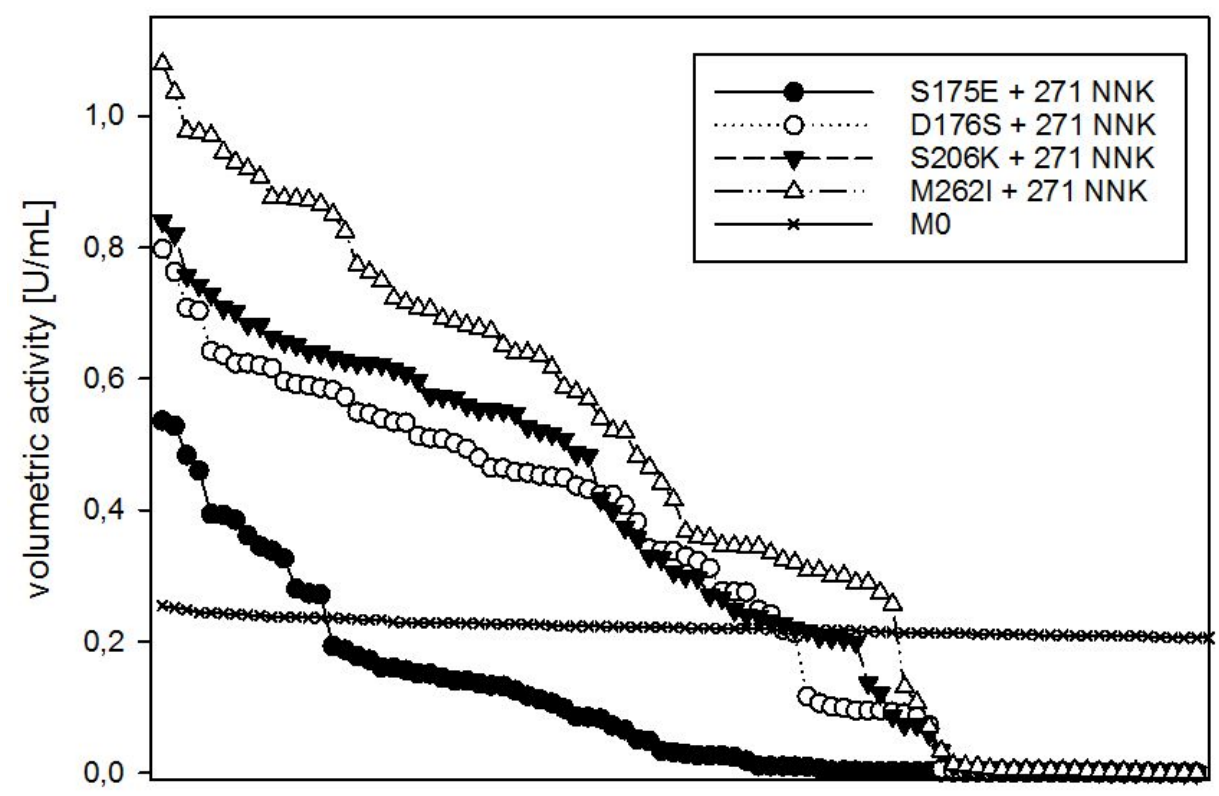

Supplemental Figure 3: Mutant landscape for single mutant in combination with 271 NNK compared to template activity (M0). The reactions were carried out at room temperature with $100 \mathrm{mM}$ HEPES pH 7.35, 100 mM ammonium sulfate $\mathrm{pH} 7,1.3 \mathrm{mM}$ DGA and $1 \mathrm{mM} \mathrm{NAD}^{+}$in a final volume of $200 \mu \mathrm{L}$.

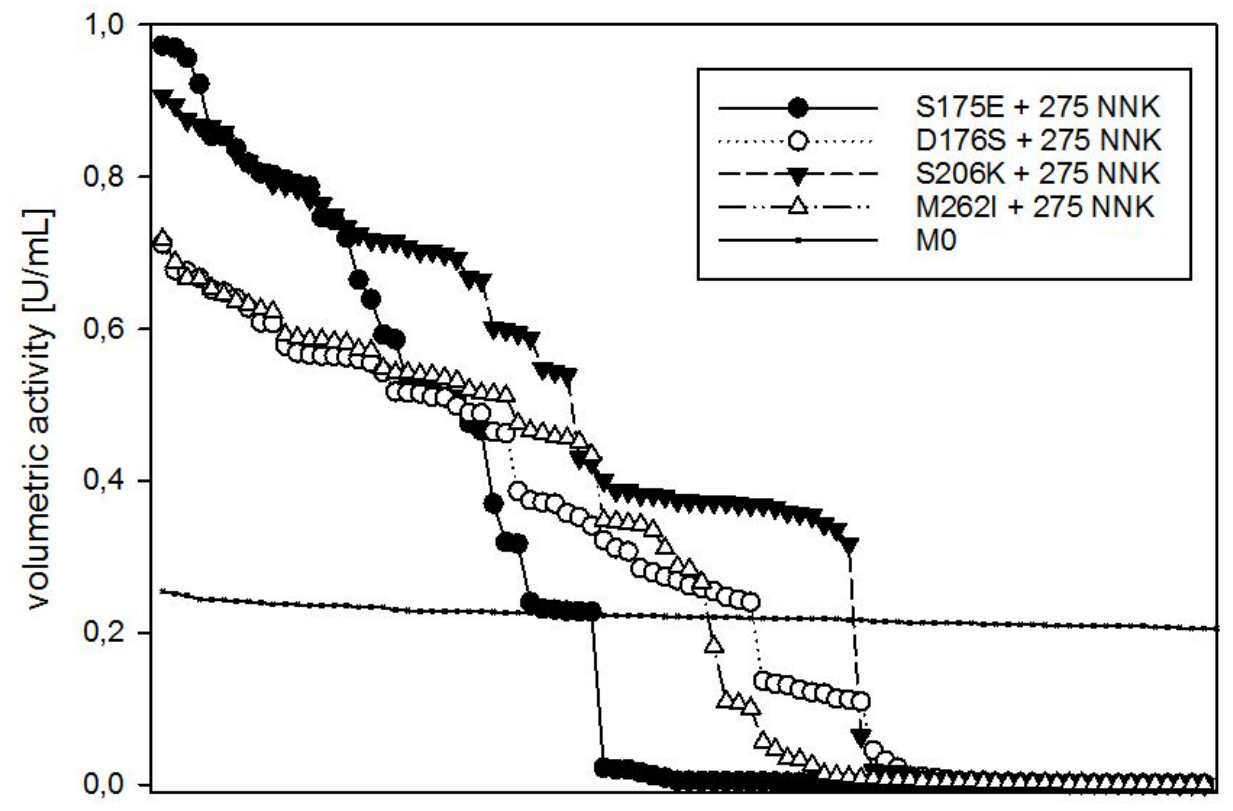

Supplemental Figure 4: Mutant landscape for single mutant in combination with 275 NNK compared to template activity (M0). The reactions were carried out at room temperature with $100 \mathrm{mM}$ HEPES pH 7.35, $100 \mathrm{mM}$ ammonium sulfate $\mathrm{pH} 7,1.3 \mathrm{mM}$ DGA and $1 \mathrm{mM} \mathrm{NAD}^{+}$in a final volume of $200 \mu \mathrm{L}$. 

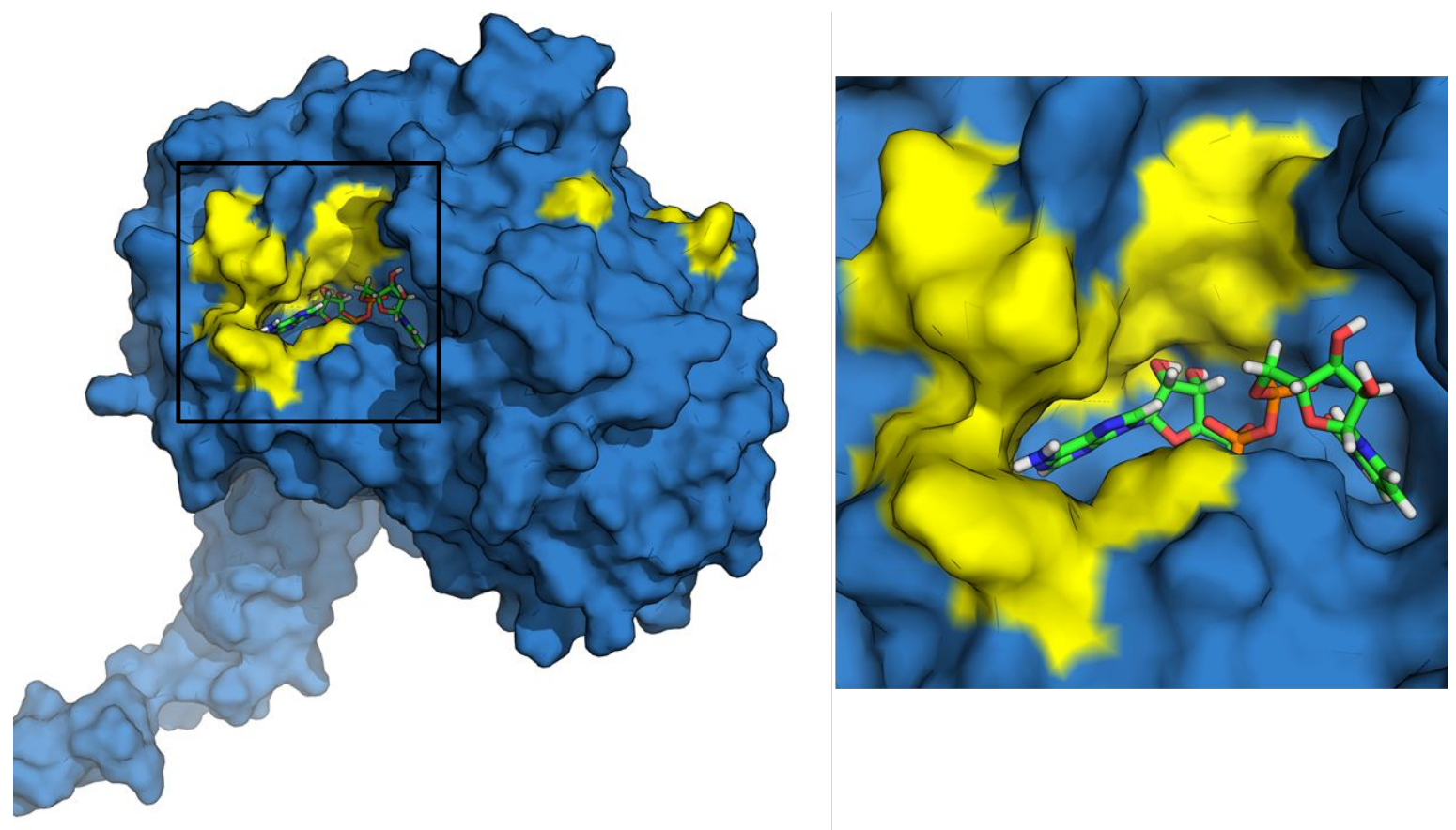

Supplemental Figure 5: 3D structure model of TaALDH (M0) with docked NAD+. Positions selected for mutations are colored in yellow. 

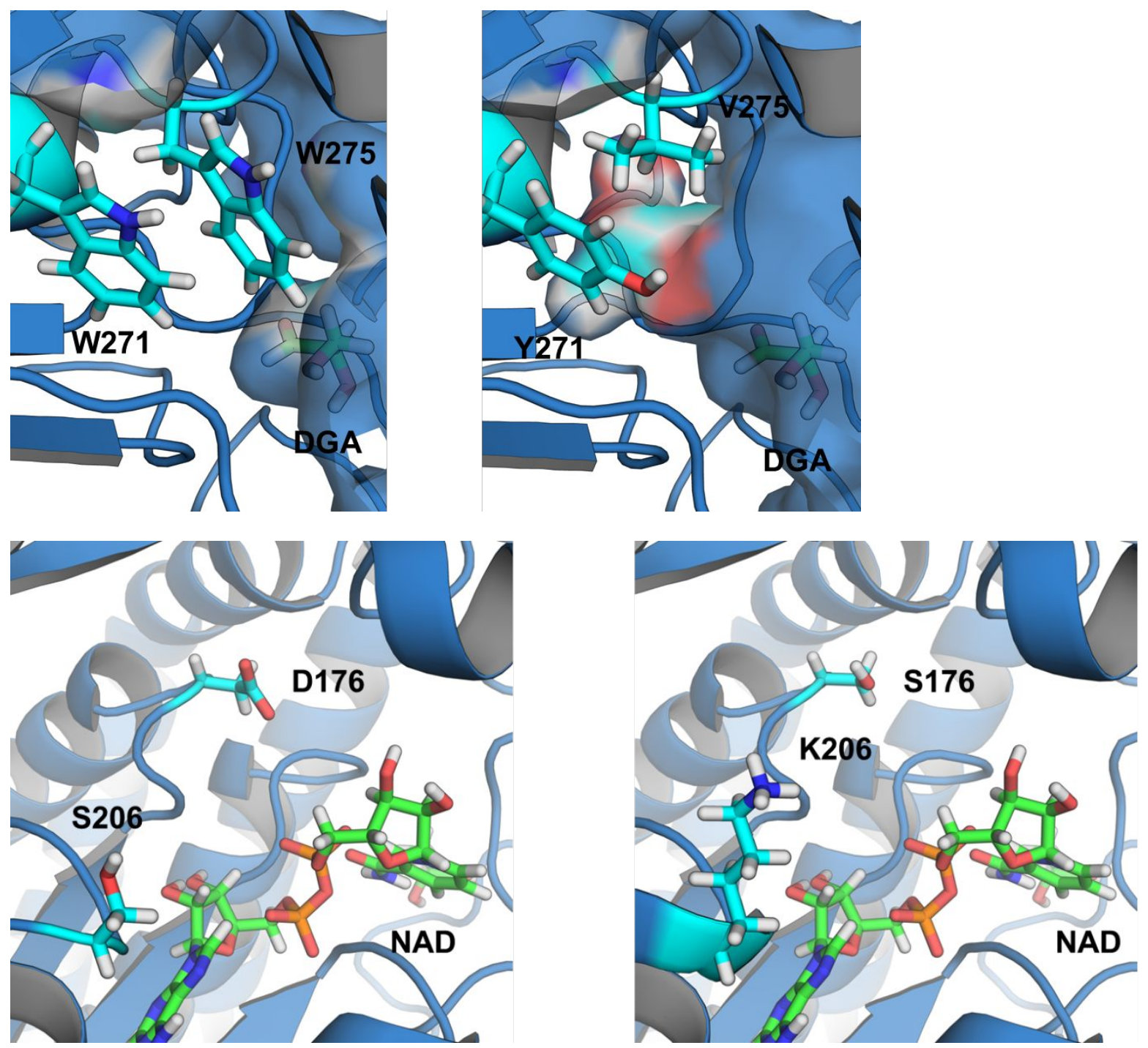

Supplemental Figure 6: Illustrative view of amino acid exchanges with the template M0 on the right side and the mutant M42 on the left. The exchanged amino acids are colored in light blue and NAD+ and DGA are in green. 


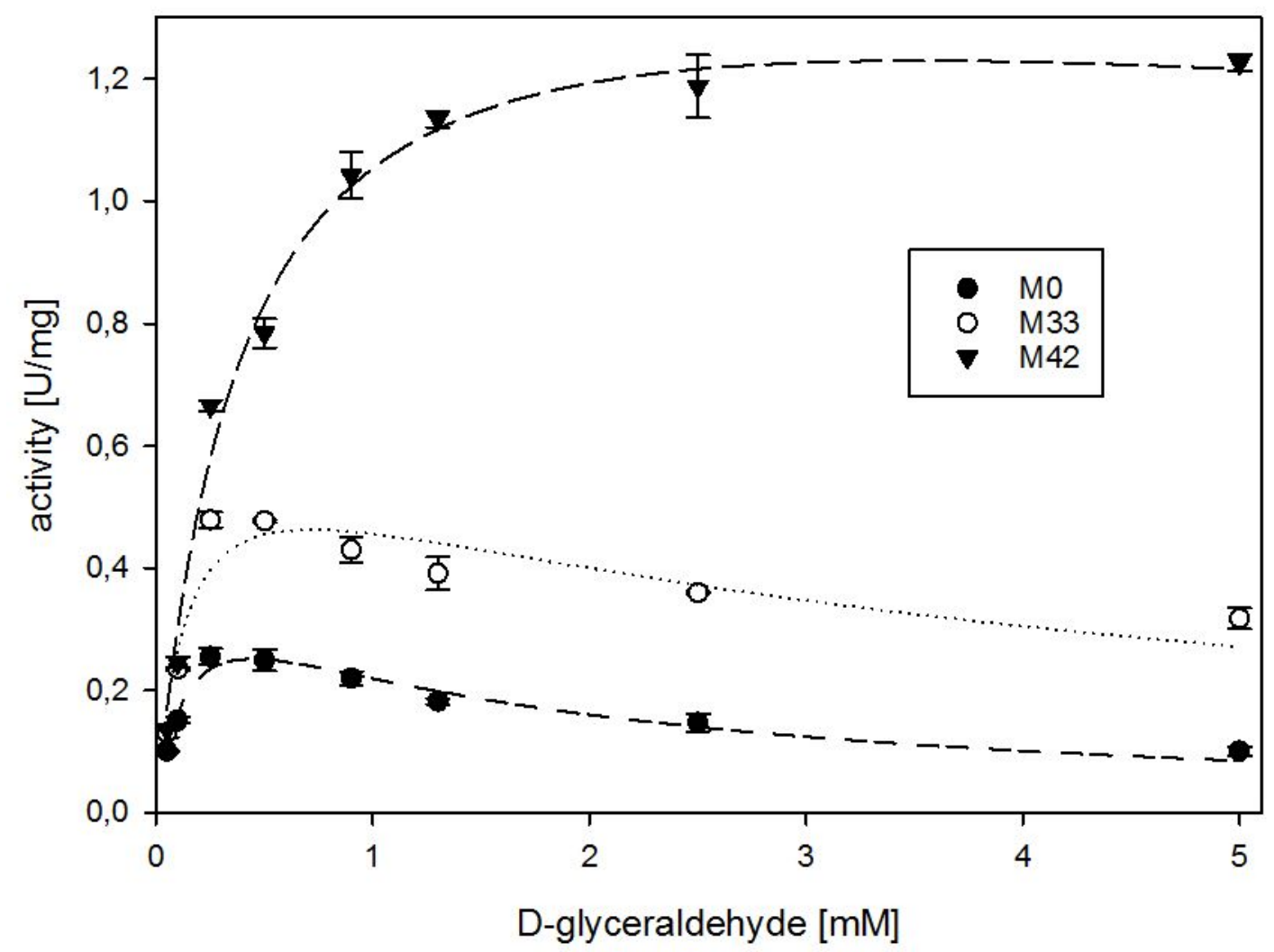

Supplemental Figure 7: Kinetic curves for template (M0) and engineered variants of TaALDH. The measurements were carried out in triplicate at $50^{\circ} \mathrm{C}$ containing $100 \mathrm{mM}$ HEPES pH 7.35 and $100 \mathrm{mM}$ ammonium sulfate $\mathrm{pH}$ 7. $\mathrm{NAD}^{+}$saturation kinetics were determined with $1.3 \mathrm{mM}$ DGA and DGA saturation measurements contained $1 \mathrm{mM} \mathrm{NAD}+$ in a final volume of $200 \mu \mathrm{L}$. 


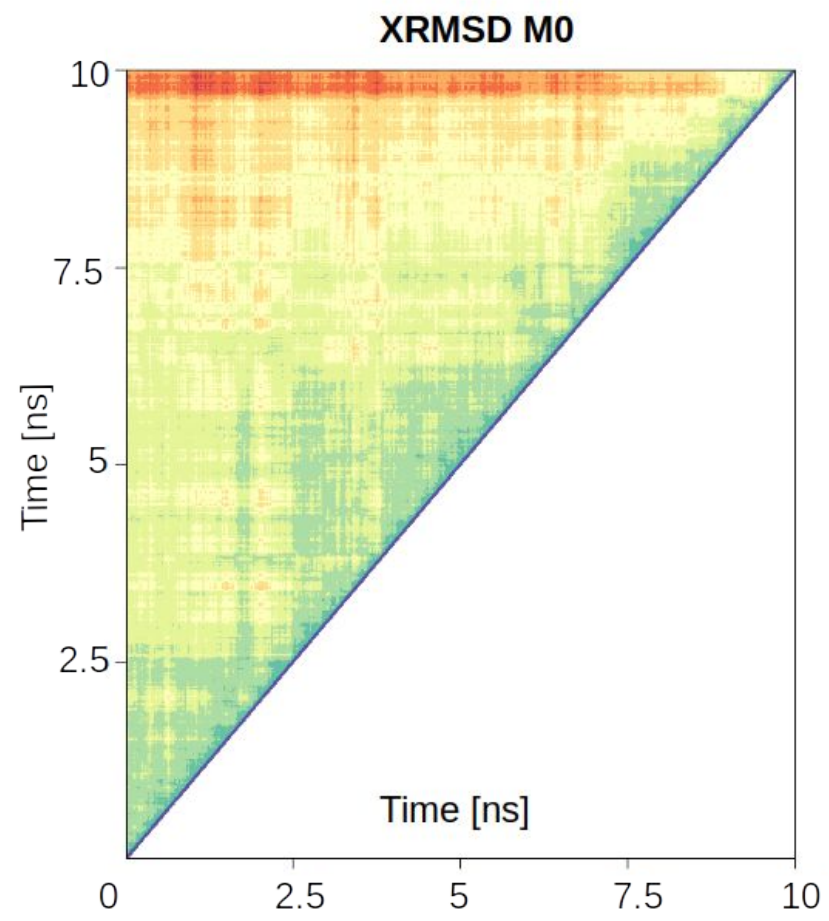

Legend [nm]

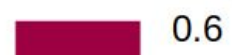

0.45

0.3

0.15

0

Supplemental Figure 8: Cross RMSD of M0 plotted via MDplot. ${ }^{5}$ For the calculations all non-hydrogen atoms were considered.

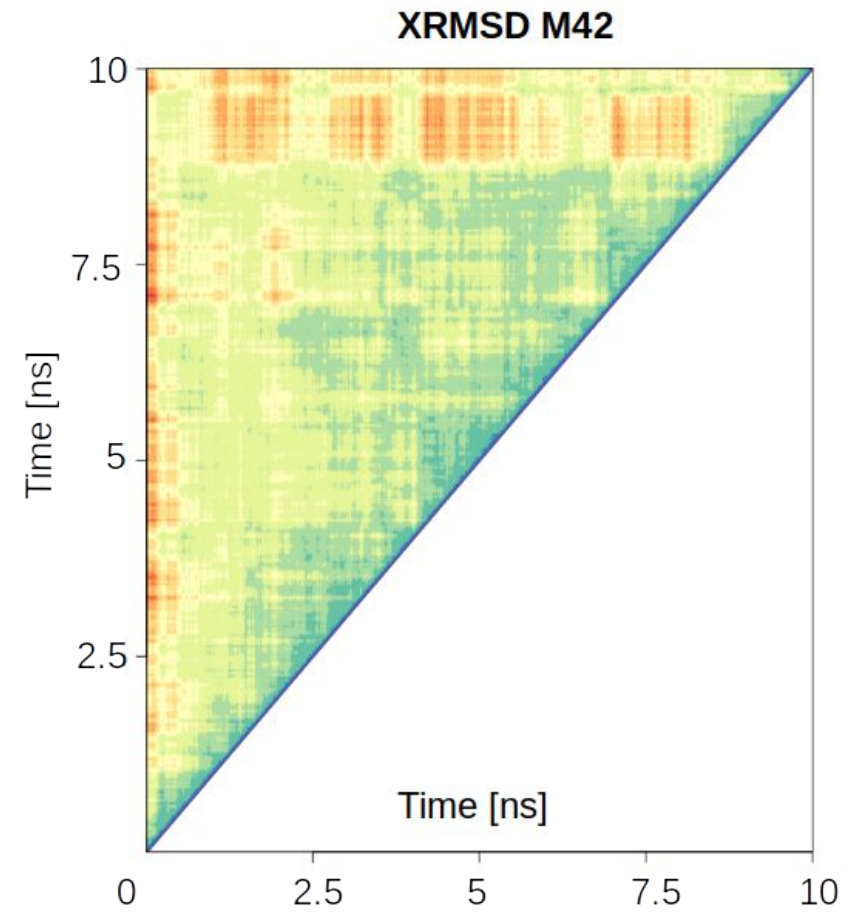

Legend [nm]

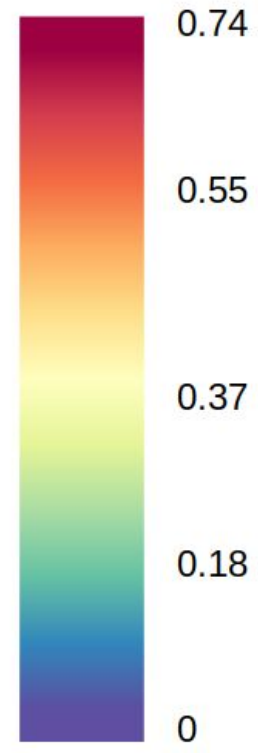

Supplemental Figure 9: Cross RMSD of M42 plotted via MDplot. ${ }^{5}$ For the calculations all non-hydrogen atoms were considered. 


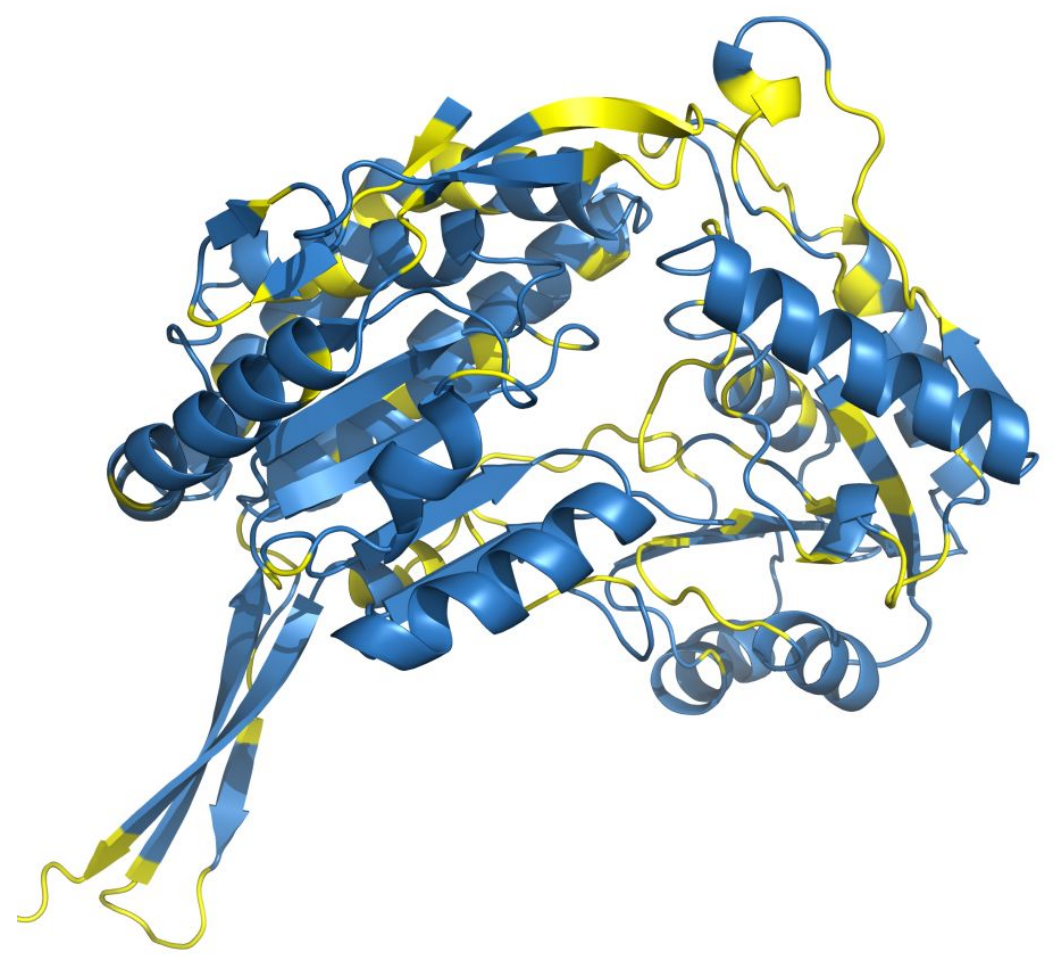

Supplemental Figure 10: Graphical visualization of the delta RMSF plot. Regions where the RMSF of M0 exceeds the RMSF of M42 are indicated in yellow.

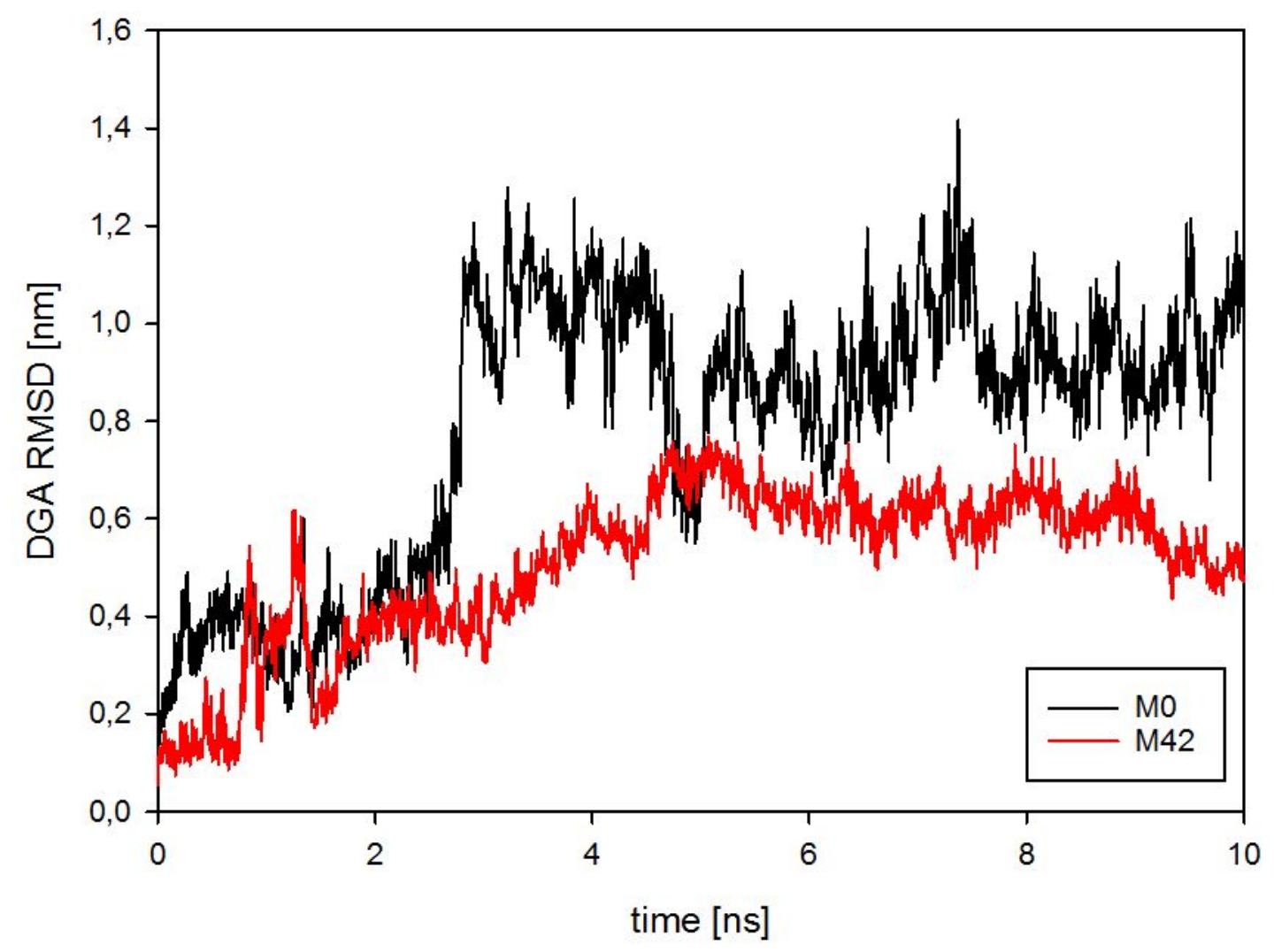

Supplemental Figure 11: RMSD of DGA in comparison to the protein backbone. 


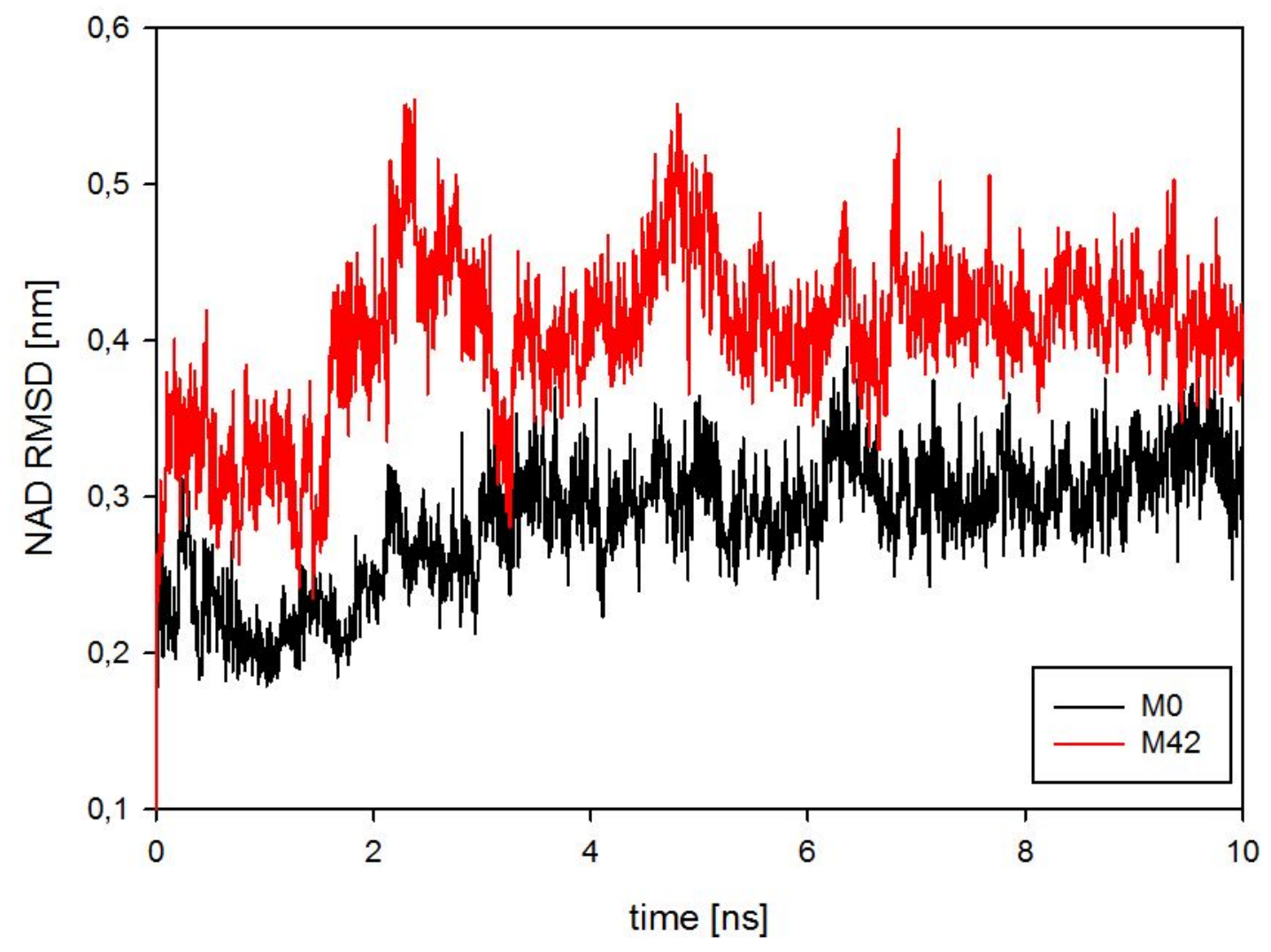

Supplemental Figure 12: RMSD of $\mathrm{NAD}^{+}$in comparison to the protein backbone.

\section{Supplemental References}

[1] Bosley, A. D., and Ostermeier, M. (2005) Mathematical expressions useful in the construction, description and evaluation of protein libraries, Biomol. Eng. 22, 57-61.

[2] Nov, Y. (2012) When second best is good enough: another probabilistic look at saturation mutagenesis, Appl. Environ. Microbiol. 78, 258-262.

[3] Cahn, J. K. B., Werlang, C. A., Baumschlager, A., Brinkmann-Chen, S., Mayo, S. L., and Arnold, F. H. (2017) A General Tool for Engineering the NAD/NADP Cofactor Preference of Oxidoreductases, ACS Synth. Biol. 6, 326-333.

[4] Koppaka, V., Thompson, D. C., Chen, Y., Ellermann, M., Nicolaou, K. C., Juvonen, R. O., Petersen, D., Deitrich, R. A., Hurley, T. D., and Vasiliou, V. (2012) Aldehyde dehydrogenase inhibitors: a comprehensive review of the pharmacology, mechanism of action, substrate specificity, and clinical application, Pharmacol. Rev. 64, 520-539.

[5] Margreitter, C., and Oostenbrink, C. (2017) MDplot: Visualise molecular dynamics, R J. 9, 164. 